\title{
The Association Between Small-for-Gestational Age Triplet Pregnancies and Neonatal Mortality: A Novel Approach to Growth Assessment in Multiple Gestations
}

\author{
ISAAC BLICKSTEIN, HAMISU M. SALIHU, LOUIS G. KEITH, AND GREG R. ALEXANDER
}

Department of Obstetrics and Gynecology [I.B.], Kaplan Medical Center, 76100 Rehovot, Israel; Hadassah-Hebrew University School of Medicine [I.B.], Jerusalem, 76100 Israel; Department of Maternal and Child Health [H.M.S., G.R.A.], University of Alabama at Birmingham, Birmingham, Alabama 35294; Department of Obstetrics and Gynecology [L.G.K.], Northwestern University, Feinberg School of Medicine, Prentice Women's Hospital and Maternity Center of the Northwestern Memorial Hospital, Chicago, Illinois 60611; The Center for Study of Multiple Birth [L.G.K.], Chicago, Illinois 60611

\begin{abstract}
It is customary to estimate the uteroplacental function in singletons by defining appropriateness of birth weight by gestational age. Such a measure, however, is not available for the entire multiple pregnancy set. We evaluate a new index, total triplet birth weight, expressed as multiples of the median (MOM) birth weight of singleton gestations. We categorized triplet sets as small-, appropriate-, and large-for-gestational age pregnancies (SGA, AGA, and LGA, respectively), defined as $<1 \mathrm{SD}, \pm 1 \mathrm{SD}$, and $>1 \mathrm{SD}$ from the mean MOM birth weight of singleton gestations. We used the 1995-1998 US matched multiple dataset to evaluate this index and to explore the association between the three categories in terms of risk of neonatal mortality. The mean $\pm \mathrm{SD}$ MOM value was $2.3 \pm 0.4$. There was an inverse correlation between mean MOM and gestational age. LGA pregnancy status was associated with multiparity, race (being white), and high social status (education). Maternal age did not influence MOM scores. Compared with the LGA pregnancy category, the risk for neonatal mortality was more than doubled in the AGA pregnancy group and more than 9-fold in the SGA pregnancy category. We propose that this new measure could be a useful proxy for the uteroplacental efficiency in a similar way that the SGA designation works for singleton infants. (Pediatr Res 59: 565-569, 2006)
\end{abstract}

$\mathrm{T}^{\mathrm{s}}$ The human female, as any other female in the animal kingdom, demonstrates an inverse relationship between litter size and both gestational duration and birth weight. Accordingly, the means of gestational ages and birth weights of triplets are $32.5 \mathrm{wk}$ and $1735 \mathrm{~g}$ compared with $39.0 \mathrm{wk}$ and $3357 \mathrm{~g}$ for singletons (1). Such plurality-dependent differences often translate to increased perinatal morbidity and mortality, especially among triplets. The 2002 American birth data indicate that the remarkable upswing in triplet and higher-order multiples of the past two decades (from 37.0 to 193.5 per 100,000 live births) appears to have reached a

Received June 17, 2005; accepted November 2, 2005.

Correspondence: Isaac Blickstein, M.D., Department of Obstetrics and Gynecology, Kaplan Medical Center, 76100 Rehovot, Israel; e-mail: blick@netvision.net.il

This study was supported in part by a grant from the Jeanette Kennelly Kroch Center for Twin Studies of the Northwestern University Memorial Foundation to the Center for Study of Multiple Births, Chicago, IL. The Flight Attendant Medical Research Institute (FAMRI) supported Dr. Salihu through a Young Clinical Scientist Award.

DOI: 10.1203/01.pdr.0000202757.47761.76 plateau, at least temporarily $(2,3)$. Nevertheless, the number of triplets born in 2002-6898 - represents a very significant number in terms of its public health implications.

The primary causes for adverse outcomes of triplets are undoubtedly the inherent increased risk of preterm birth and low birth weight. Alexander et al. (1) showed that as many as $32 \%$ of triplets are VLBW $(<1500 \mathrm{~g})$ compared with $1 \%$ of singletons, and as many as $41 \%$ of triplets are born very preterm ( $<33 \mathrm{wk}$ ) compared with $1.7 \%$ of singletons. Moreover, the incidence of triplets among the cohort of extremely low birth weight infants $(<1000 \mathrm{~g})$ is 10 times higher than that in the general population (4).

It is currently believed that the in utero growth pattern of triplets parallels that of singletons until about 28 wk gestation but changes into growth deceleration thereafter (5). Individual triplets, therefore, exhibit relative growth restriction compared with singletons throughout the third trimester $(5,6)$. At the same time, however, the uterine milieu in mothers of triplets displays a remarkable adaptation to the multiple gestation and is operating in full capacity. For example, based on the analysis of 5172 triplet sets, it was found that, as early as $25 \mathrm{wk}$ gestation, the total triplet birth weight exceeds that of the 90th birth weight percentile for singletons born near term, and, at $29 \mathrm{wk}$, it exceeds the median birth weight of singletons at $40 \mathrm{wk}$ gestation (5). All the above point to the concept that at the same time that the entire multiple pregnancy is growth promoted compared with a singleton gestation, the individual fetus in a multiple pregnancy is likely to be growth restricted (5). Therefore, estimating uterine efficiency to nurture the entire multiple pregnancy rather than the individual babies appears to be effective as a measure of overall well being of the multiple gestation.

\footnotetext{
Abbreviations: AGA, appropriate for gestational age; AOR, adjusted odds ratio; LGA, large for gestational age; MOM, multiples of the median; SGA, small for gestational age; VLBW, very low birth weight
} 
The uterine effectiveness to nurture singletons can be inferred by assigning a given birth weight (estimated or actual) within the distribution of birth weights (i.e. birth weight percentile). Normally growing singletons demonstrate an almost linear relationship between birth weight and gestational age at birth. Because of the limited uterine size, however, this desirable growth is not possible for triplets. Moreover, in triplets, wherein each sib may have its own growth pattern (6), it is not possible to estimate the efficiency of the entire uterine environment in terms of adapting to the triplet gestation and in nurturing the entire set. Simply stated, whereas the function of the uteroplacental unit is usually estimated by proxy, designating a given neonate as SGA, such a measure for the entire triplet pregnancy set is not available. The designation of SGA in a triplet pregnancy could be as useful as the designation of SGA is in singletons. Moreover, if such an antenatal diagnosis were available, it could be useful to detect the important transition from uterine adaptation to uterine exhaustion-a key point in clinical management of triplet gestations $(5,6)$.

In this study, we used a new approach, defining the entire fetal mass of a triplet set in terms of MOM birth weight of singletons of the same gestational age. This is a proxy measure to define the degree to which the uterine environment is performing in a multiple compared with the average singleton gestation. We then used an epidemiologic approach to evaluate the association between this surrogate means of uterine efficacy and neonatal mortality in triplets.

\section{METHODS}

First, we established the median birth weight of singletons delivered between 24 and 38 wk gestation, using the 1995-1998 linked birth/infant death data set (7), available from the National Center for Health Statistics and comprising over 15 million singleton births. Subsequently, we used data from the 1995-1998 Matched Multiple Birth Dataset, also available from the same source (8). Of a total of 6710 triplet maternities, we studied 6646 live-born sets delivered between 24 and 38 wk gestation. The 64 exclusions (approximately $1.0 \%$ ) were sets with one or more stillbirth or those with spurious results [according to the method of Kiely et al. (9)]. We calculated the total triplet birth weight, and expressed this value as MOM birth weight for singletons born at the same gestational age derived from the National Center for Health Statistics database mentioned above. We then established the normality of the distribution of MOM by the Shapiro-Wilk method. This step was necessary because the lower and upper tails (i.e. below and above $1 \mathrm{SD}$ ) are only appropriate for analysis in normal distributions. The analysis indicated an essentially normal distribution (skewness 0.3 and kurtosis of 1.0). The mean \pm 1 SD MOM value was $2.3 \pm 0.4$. By using \pm 1 SD as an arbitrary value, we defined three groups of triplet pregnancies: low total fetal mass or SGA (MOM $<1$ SD below the mean MOM of singleton birth weight), average total fetal mass or AGA (MOM within the mean MOM $\pm 1 \mathrm{SD}$ ), and high total fetal mass or LGA (MOM $>1$ SD above the mean MOM of singleton birth weight).

In our analysis, we compared the following variables in the three total fetal mass groups: maternal age (mean, $<30,30-35$, and $>35 \mathrm{y}$ ), frequency of nulliparas, gestational age (mean, $<28,28-32,33-35$, and $>35 \mathrm{wk}$ ), and neonatal mortality (at $\leq 28 \mathrm{~d}$ postpartum). Maternal and gestational age categories were chosen because previous studies found these subgroups as being of prime importance in triplet gestations (10).

We used the $t$ and the $\chi^{2}$ tests for continuous and categorical variables, respectively. Where expected cell size was $<5$, Fisher's exact test was applied. The strength of the relationship between frequencies was measured by Pearson's coefficient ( $R$ value) and tested for significance against a zero slope. In this type of analysis, a higher correlation is expected with means than with individual entries. We also assessed the relationship between total triplet mass group and risk of neonatal mortality using a multivariable logistic regression model, adjusting for intracluster correlations by means of the generalized estimating equations (11). A standardized probability curve to predict neonatal mortality independent of gestational age was then constructed based on parameter estimates for MOM score. This was obtained using the following formula (12): $e^{\beta \mathrm{x}} / 1+e^{\beta \mathrm{x}}$, where $e^{\beta \mathrm{x}}$ is the adjusted odds ratio for neonatal mortality for a given MOM score.

For statistical analysis, we used a SAS/STAT statistical package (version 8, Cary, NC) and True Epistat Software (Math Archives, Round Rock, TX). $P$ values $<0.05$ were considered significant. The Institutional Review Board of the University of Alabama at Birmingham approved this study.

\section{RESULTS}

The average MOM values of the triplet sets decreased as a highly significant, inversely linear function of gestational age $\left(\mathrm{MOM}=4.6-0.06 \mathrm{wk}, R^{2}=0.95\right)$. Figure 1 shows the trend lines for the mean MOM $\pm 1 \mathrm{SD}$. Sets with total birth weight above 3 MOM (i.e. thrice the singleton's median birth weight) were born mainly before $28 \mathrm{wk}$, sets with total birth weight below 2 MOM (i.e. twice the singleton's median birth weight) were born mainly after $32 \mathrm{wk}$, and sets with average MOM were always between 2 and 3 times the median birth weight of a singleton at the same gestational age. When the average MOM values by gestational age were compared between the 4375 primiparas and 2243 multiparas (in 28 sets or $0.4 \%$, parity status was missing), both trend lines exhibited a significant correlation between average MOM and gestational age $\left(R^{2}=0.88\right.$ and 0.93 , respectively), but have a significantly different slope $[p=0.04,95 \%$ confidence interval (CI) for the slope $-0.06,-0.05]$. The different inclinations represent significantly lower mean MOM among primiparas, consistently found in the comparison of mean MOM for each gestational age between 32 and 36 wk gestation (all $p$ values $<0.001)$. Comparison between the frequencies of primiparas among the three groups shows a gradual but significant decline, whereby fewer nulliparous mothers were found among AGA compared with LGA pregnancies, and among AGA compared with SGA pregnancies (Table 1).

Table 1 further shows that being nonwhite and having $<12$ y of schooling was significantly associated with lower total fetal mass. As expected from Figure 1, LGA pregnancies

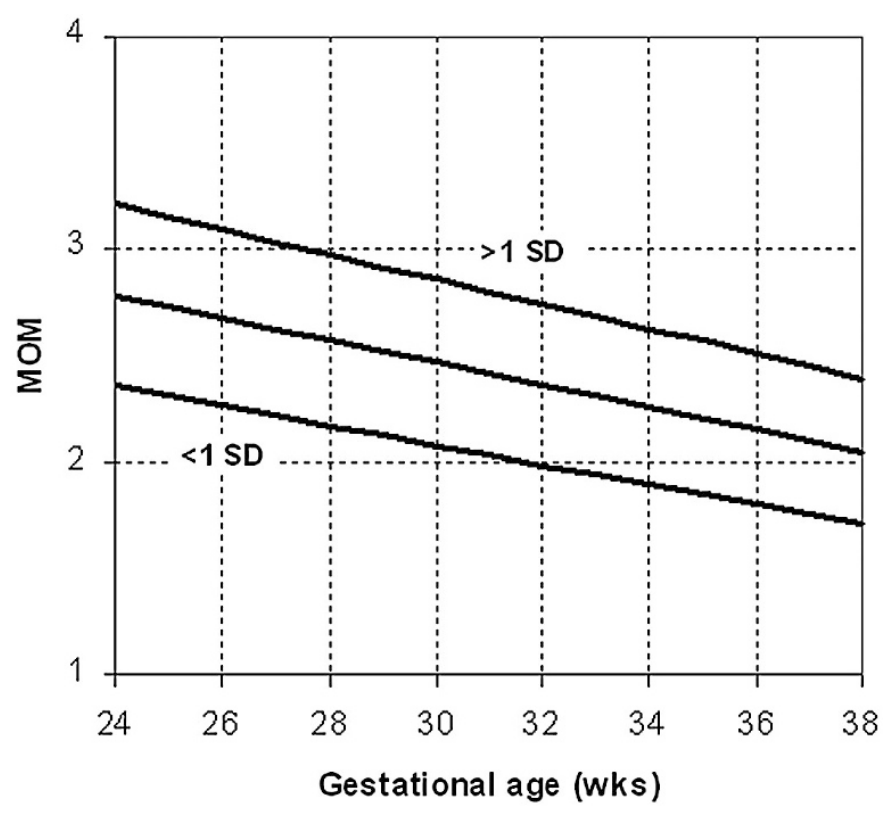

Figure 1. Trend lines for the mean $\mathrm{MOM} \pm 1 \mathrm{SD}$ by gestational week. 
Table 1. Comparison of demographic characteristics between SGA, AGA, and LGA triplet pregnancies

\begin{tabular}{|c|c|c|c|c|}
\hline \multirow[b]{2}{*}{ No. } & \multicolumn{3}{|c|}{ Triplet pregnancies } & \multirow[b]{2}{*}{ Statistics } \\
\hline & 1049 & 4897 & 700 & \\
\hline Primiparas & $636(60.6)^{*}, \ddagger$ & $3229(65.9) \dagger, \ddagger$ & $510(72.9)^{*}, \dagger$ & $\begin{array}{l}* 1.7(1.4,2.1) \\
\dagger 1.4(1.1,1.7) \\
\ddagger 1.2(1.1,1.4)\end{array}$ \\
\hline \multicolumn{5}{|l|}{ Maternal age (y) } \\
\hline Mean & $32.0 \pm 5.0$ & $31.8 \pm 4.9$ & $31.2 \pm 5.5$ & \\
\hline$<30$ & $310(29.5)$ & $1481(30.3)$ & $236(33.7)$ & \\
\hline $30-35$ & $432(41.2)$ & $2048(41.8)$ & $278(39.7)$ & \\
\hline$>35$ & $307(29.3)$ & $1368(27.9)$ & $186(26.6)$ & \\
\hline \multicolumn{5}{|l|}{ Gestational age (wk) } \\
\hline
\end{tabular}

Data are shown as number (\%). Statistics are shown as odds ratio (OR) (95\% CI) and as $p$ value for categorical and continuous variables, respectively.

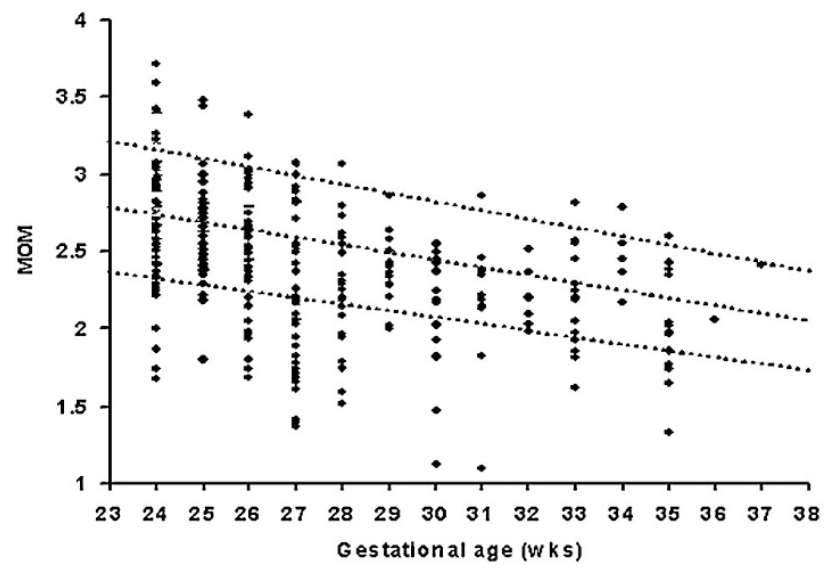

Figure 2. Neonatal deaths by gestational age. Trend lines for the mean MOM $\pm 1 \mathrm{SD}$ are superimposed.

Table 2. Neonatal mortality (rate per 1000 live-born infants) by total fetal mass group and across gestational age strata

\begin{tabular}{|c|c|c|c|c|c|c|c|}
\hline & \multicolumn{6}{|c|}{ Triplet pregnancies } & \multirow[b]{3}{*}{ Statistics } \\
\hline & \multicolumn{2}{|l|}{ LGA } & \multicolumn{2}{|l|}{ AGA } & \multicolumn{2}{|l|}{ SGA } & \\
\hline & $N=3,147$ & Rate & $N=14,691$ & Rate & $N=2,100$ & Rate & \\
\hline$<28$ wk & $100 / 776$ & 128.9 & $162 / 727$ & 222.8 & $37 / 68$ & 544.1 & $\begin{array}{l}<0.001^{*} \\
<0.001 \dagger\end{array}$ \\
\hline $28-32 \mathrm{wk}$ & $6 / 1417$ & 4.2 & $66 / 5082$ & 13.0 & $15 / 647$ & 23.3 & $\begin{array}{r}0.005^{*} \\
<0.001 \dagger\end{array}$ \\
\hline $33-35 \mathrm{wk}$ & $2 / 761$ & 2.6 & $22 / 7007$ & 3.1 & $11 / 942$ & 11.7 & $\begin{array}{l}0.8^{*} \\
0.03 \dagger\end{array}$ \\
\hline$>35 \mathrm{wk}$ & $0 / 193$ & 0.0 & $3 / 1875$ & 1.6 & $1 / 445$ & 2.2 & $\begin{array}{l}0.7 * \\
0.5 \dagger\end{array}$ \\
\hline
\end{tabular}

Figures are shown as $n / N$; statistics are shown as $p$ value.

* Differences in neonatal mortality rate between LGA and AGA pregnancies.

$\dagger$ Differences in neonatal mortality rate between LGA and SGA pregnancies.

were associated with significantly lower gestational age, mainly at $<28$ wk $(\mathrm{OR}=11.0 ; 95 \% \mathrm{CI}, 6.8,18.1)$, and, conversely, SGA pregnancies were associated with signifi-

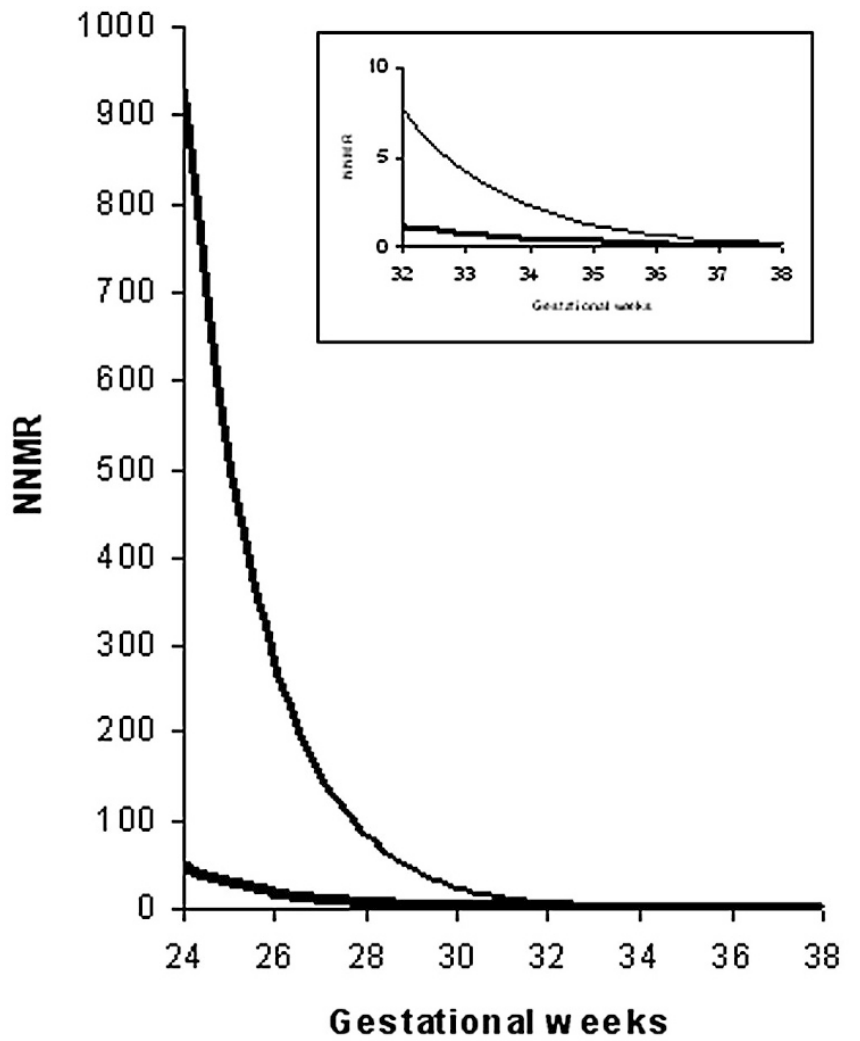

Figure 3. Neonatal death rate (per 1000 live born) among SGA (upper curve) and LGA (lower curve) triplet pregnancies. Insert shows the curves at $>32$ wk.

cantly more advanced gestational age, mainly at $>35 \mathrm{wk}$ gestation $(\mathrm{OR}=4.6$; 95\% CI, 3.3, 6.3). Maternal age subgroups, however, were not associated with any of the total fetal mass groups (all OR include 1).

There were 425 neonatal deaths among the 19,938 live-born neonates (21.3/1000, Fig. 2). Because of the strong association between total fetal mass group and gestational age, we present the neonatal mortality rates according to four gestational 
Table 3. Neonatal mortality estimates by uterine efficacy status using the generalized estimating equation

\begin{tabular}{cccc}
\hline $\begin{array}{c}\text { Triplet } \\
\text { pregnancies }\end{array}$ & AOR & $95 \%$ CI & $p$ Value \\
\hline LGA & 1.0 & - & - \\
AGA & 2.4 & $1.8-3.2$ & $<0.0001$ \\
SGA & 9.2 & $5.9-14.4$ & $<0.0001$ \\
\hline
\end{tabular}

Adjusted estimates were generated after controlling for the confounding effects of parity, race, educational status, and gestational age.

age-specific strata (Table 2). The frequencies of neonatal deaths decrease with gestational age in a perfect exponential function for all three groups (Fig. 3); however, it is clear that neonatal deaths occur more frequently among SGA triplet pregnancies (Fig. 2 and Table 2). Overall, the neonatal death rate among triplets in the SGA pregnancies group was significantly higher than that of the AGA pregnancies $(\mathrm{OR}=3.0$; $95 \% \mathrm{CI}, 2.2,4.1)$ and the LGA pregnancies $(\mathrm{OR}=6.8 ; 95 \%$ CI, 4.3, 10.8) groups. Moreover, the neonatal deaths among the AGA pregnancies were also higher compared with LGA pregnancies (OR $=2.0 ; 95 \% \mathrm{CI}, 1.6,2.6)$. In fact, after $28 \mathrm{wk}$, frequency of death among LGA triplet pregnancies was extremely low (8/2371 or 3.4/1000 live-born infants). These results were confirmed even after adjusting for confounding characteristics and correcting for the bias effect of intracluster correlations by applying the generalized estimating equations framework (Table 3).

\section{DISCUSSION}

This study examines a new approach to determine the uterine potential to sustain the excess of fetal mass in a multiple gestation. The assumption behind this model is that the entire triplet pregnancy should ideally weigh thrice the birth weight of an average singleton of the same gestational age. However, in the overwhelmed uterine environment, this potential is reduced, so that the entire fetal mass of the multiple gestation is diminished compared with what would have been expected.

By using the total triplet mass defined as MOM of the singleton birth weight, the entire pregnancy rather than individual fetal growth is considered. This measure was chosen because it bears not only a natural significant correlation with individual fetal growth curves (6) but also a significant correlation with discordant fetal growth in triplets (13). For example, the latter association was studied in a sample of nearly 800 triplet sets with a birth weight discordance of more than $25 \%$ between the larger and the smaller sibs, and exhibited an almost perfect polynomial relationship $\left(R^{2}=0.97\right)$ with total triplets birth weight (13). The MOM value was also chosen because it reflects the holistic approach toward the multiple pregnancy, its inherent simplicity, and because it avoids using other measures of location such as percentiles or three individual fetal weights.

We used a large, population-based data set of liveborn triplets to test this model, first, by comparing the findings to existing data and, second, by evaluating the model in terms of neonatal mortality. We note the inherent limitation of any administrative or retrospective database with its acknowledged potential of poor dating. However, this limitation is partially compensated by the large sample size of our series.

In the first step of the analysis, we showed that growth of the entire triplet pregnancy decreased in a predictable manner compared with the average singleton. This finding corresponds to existing birth weight by gestational age curves (so-called growth curves) that show that growth of triplets is not different from that of twins and singletons until $28 \mathrm{wk}$, and is not different from that of twins until between 28 and $32 \mathrm{wk}$ $(1,5)$. We also showed that the model for triplets is parity dependent, in agreement with repeated studies documenting that the parous uterus is more effective to nurture multiples $(5,6,14)$. Moreover, low total triplet weight was influenced by race and education, as was found by others (15). As a result, more triplet sets are delivered at lower gestational ages from very "efficient" uterine milieus simply because there was not enough time to demonstrate the phenomenon of reduced "efficiency." Conversely, at higher gestational ages, there was sufficient time to exhibit the full range of uterine capability to nurture triplets and to demonstrate its decreased potential to produce sets that weighed thrice the median birth weight of same-age singletons.

In the second step, we evaluated the association of neonatal mortality with the three different total fetal mass groups. Not unexpectedly, despite higher uterine effectiveness, extreme preterm births $(<28 \mathrm{wk})$ were associated with much higher neonatal death rates. However, our data clearly show that SGA pregnancies are always associated with highest neonatal mortality, whereas LGA pregnancies are always associated with the lowest neonatal mortality rate (Table 2).

Figure 1 suggests that high MOM values of the total triplet weight at advanced gestational age are not to be expected. For instance, a value of $2.5 \mathrm{MOM}$ birth weight of singletons $(>1 \mathrm{SD}$ above the mean MOM) at $38 \mathrm{wk}$ is lower than the average MOM before $28 \mathrm{wk}$ gestation. In simple terms, it means that the payoff to achieve an advanced gestational age is reduction in uterine ability to produce $3 \mathrm{MOM}$ of the singleton birth weight. At the same time, however, it should be kept in mind that each member of the triplet set, as well as the entire set, continues to grow (6), and a value of 1.8 MOM birth weight of singletons ( $<1 \mathrm{SD}$ below the mean MOM) at $38 \mathrm{wk}$ is still $80 \%$ more fetal mass compared with the average singleton. It seems, therefore, that the reduced "efficacy" of the uterine environment has a definite physiologic purpose- to increase maturity in expense of fetal size.

Looking at Figure 2, one may wonder whether higher efficacies at lower gestational ages are advantageous at all. As may well be the case, reaching three times the median birth weight of a singleton by 28 wk may represent the maximal capacity for the uterine milieu for as many as $25.7 \%$ of the sets. In these instances, preterm deliveries with appropriately grown triplets will ensue, merely because the uterus has not been able to adapt to the multiple pregnancy.

All the above observations neatly translate in the neonatal mortality figures. In all probability, and at all gestational ages, the highly efficacious uterus is associated with fewer neonatal deaths. The correlation between neonatal mortality and the 
MOM model proposed in this study suggests that this new approach can be used also to correlate the MOM model with perinatal morbidity. Based on these findings, additional studies should be conducted to identify maternal characteristics that are associated with LGA triplet pregnancies and lower neonatal mortality. Such characteristics may identify a maternal phenotype that is likely to have either a favorable or an adverse outcome of their triplet gestation. Such information might become a key variable in counseling couples contemplating multifetal pregnancy reduction. Moreover, the recognition of an inefficient uterine milieu during pregnancy may lead to a timely referral to a tertiary medical center, where the triplet neonates of a growth-restricted pregnancy may receive appropriate intensive neonatal care.

Finally, the potential clinical application of the findings in this study merits emphasis. Generally, we studied SGA triplet sets rather than SGA infants in a triplet set. Currently, this method was applied to evaluate the relationship with neonatal mortality, and although it may not change the management of triplet neonates, it nevertheless might explain why certain triplet pregnancies, within a given gestational age, are more at risk of neonatal death. Obviously, if this information is available during pregnancy, it may indicate that the uterine environment is unable to further maintain the entire triplet pregnancy rather than an individual fetus. This stage of pregnancy, referred to as the transition from uterine adaptation to uterine exhaustion $(5,6)$, may indicate a closer follow up of these pregnancies and, perhaps, earlier intervention.

\section{REFERENCES}

1. Alexander GR, Kogan M, Martin J, Papiernik E 1998 What are the fetal growth patterns of singletons, twins, and triplets in the United States? Clin Obstet Gynecol 41:114-125

2. Martin JA, Hamilton BE, Sutton PD, Ventura SJ, Menacker F, Munson ML 2003 Births: final data for 2002. Natl Vital Stat Rep 52:1-113

3. Blickstein I, Keith LG 2005 The decreased rates of triplet births: temporal trends and biologic speculations. Am J Obstet Gynecol 193:327-331

4. Vohr BR, Wright LL, Dusick AM, Mele L, Verter J, Steichen JJ, Simon NP, Wilson DC, Broyles S, Bauer CR, Delaney-Black V, Yolton KA, Fleisher BE, Papile LA, Kaplan MD 2000 Neurodevelopmental and functional outcomes of extremely low birth weight infants in the National Institute of Child Health and Human Development Neonatal Research Network, 1993-1994. Pediatrics 105:1216-1226

5. Blickstein I 2002 Normal and abnormal growth of multiples. Semin Neonatol 7:177-185

6. Blickstein I, Jacques DL, Keith LG 2002 Total and individual triplet birth weights as a function of gestational age. Am J Obstet Gynecol 186:1372-1375

7. National Center for Health Statistics 1995-1999 Linked Birth/Infant Death Data Set US Department of Health and Human Services, Centers for Disease Control and Prevention, Hyattsville, MD, NCHS CD-ROM Series 20, Nos. 12a, 14a-17a

8. Martin J, Curtin S, Saulnier M, Mousavi J 2003 Development of the matched multiple birth file. In: 1995-1998 Matched Multiple Birth Dataset. National Center for Health Statistics, Hyattsville, MD, NCHS CD-ROM series 21, no. 13a

9. Kiely JL 1998 What is the population-based risk of preterm birth among twins and other multiples? Clin Obstet Gynecol 41:3-11

10. Keith LG, Goldman RD, Breborowicz G, Blickstein I 2004 Triplet pregnancies in women aged 40 or older: a matched control study. J Reprod Med 49:683-688

11. Zeger SL, Liang KY 1986 Longitudinal data analysis for discrete and continuous outcomes. Biometrics 42:121-130

12. Clayton D, Hills M 1993 Statistical Models in Epidemiology. Oxford University Press, Oxford, UK

13. Blickstein I, Jacques DL, Keith LG 2003 A novel approach to intertriplet birth weight discordance. Am J Obstet Gynecol 188:1026-1030

14. Salihu HM, Aliyu MH, Rouse DJ, Kirby RS, Alexander GR 2004 The association of parity with mortality outcomes among triplets. Am J Obstet Gynecol 190:784-789

15. Zhang J, Meikle S, Grainger DA, Trumble A 2002 Multifetal pregnancy in older women and perinatal outcome. Fertil Steril 78:562-568 